Revue musicale OICRM

\title{
Internationalisme, cosmopolitisme («Anthologie du PHEM » / Mots clés, 1)
}

\section{Judy-Ann Desrosiers et Federico Lazzaro}

Volume 4, numéro 2, 2017

URI : https://id.erudit.org/iderudit/1043228ar

DOI : https://doi.org/10.7202/1043228ar

Aller au sommaire du numéro

\section{Éditeur(s)}

Observatoire interdisciplinaire de création et recherche en musique (OICRM)

\section{ISSN}

2368-7061 (numérique)

Découvrir la revue

\section{Citer cet article}

Desrosiers, J.-A. \& Lazzaro, F. (2017). Internationalisme, cosmopolitisme ("Anthologie du PHEM » / Mots clés, 1). Revue musicale OICRM, 4(2), 189-215. https://doi.org/10.7202/1043228ar
Résumé de l'article

Ce premier chapitre de la section Mots clés de la série "Anthologie du PHEM " s'intéresse à une des questions cruciales du débat musicographique parisien dans l'entre-deux-guerres, à savoir les rapports entre musique et internationalisme (ou cosmopolitisme). Les trois articles sélectionnés expriment trois positions très différentes et articulées : l'universalisme francocentrique, la condamnation de l'internationalisme considéré comme illusoire et l'utopie cosmopolite. À une époque traversée par l'idéal d'une coopération internationale pacifique et par les nationalismes les plus exacerbés, les discours développés dans ces articles permettent de faire la lumière sur les enjeux politiques et esthétiques qui sous-tendent le débat musical. 


\title{
Internationalisme, cosmopolitisme ( Anthologie du PHEM » / Mots clés, 1)
}

\author{
Judy-Ann Desrosiers et Federico Lazzaro
}

\begin{abstract}
Résumé
Ce premier chapitre de la section Mots clés de la série "Anthologie du PHEM " s'intéresse à une des questions cruciales du débat musicographique parisien dans l'entre-deux-guerres, à savoir les rapports entre musique et internationalisme (ou cosmopolitisme). Les trois articles sélectionnés expriment trois positions très différentes et articulées : l'universalisme francocentrique, la condamnation de l'internationalisme considéré comme illusoire et l'utopie cosmopolite. À une époque traversée par l'idéal d'une coopération internationale pacifique et par les nationalismes les plus exacerbés, les discours développés dans ces articles permettent de faire la lumière sur les enjeux politiques et esthétiques qui sous-tendent le débat musical.

Mots clés : cosmopolitisme ; esthétique musicale ; internationalisme ; nationalisme ; presse musicale.
\end{abstract}

\begin{abstract}
This first chapter of the Keyword section of the collection "Anthologie du PHEM" (PHEM's source readings) addresses the relationship between music and internationalism (or cosmopolitanism), that is one of the most crucial issues arising from the Parisian musical debate of the interwar period. The three selected articles defend three different positions: a francocentric universalism, the condemnation of internationalism considered as illusory, and the utopia of cosmopolitanism. In the interwar period, the ideal of a pacific international cooperation coexisted with the most extreme nationalisms, and the discourse presented in this articles provides a deeper insight into the political and aesthetical issues behind the musical debate.

Keywords: aesthetics of music; cosmopolitanism; internationalism; musical press; nationalism.
\end{abstract}


En ouverture de la série "Anthologie du PHEM » (section " Mots clés »), nous publions trois textes qui abordent une des questions cruciales du débat musicographique parisien durant l'entre-deux-guerres, à savoir les rapports entre musique et internationalisme (ou cosmopolitisme). À une époque où l'utopie d'un monde pacifié et enrichi par la coopération internationale côtoie la montée des nationalismes les plus haineux, plusieurs interventions dans la presse musicale (des phrases isolées autant que des articles spécifiques) témoignent des positionnements des musicographes dans le continuum des idées sur les échanges musicaux entre les nations. Les trois articles sélectionnés expriment trois positions très différentes et articulées : 1'universalisme francocentrique, la condamnation de l'internationalisme considéré comme illusoire et l'utopie cosmopolite.

Dans sa conférence de 1938 intitulée "Les caractéristiques de la musique française ", Robert Bernard exprime une position fortement nationaliste et " francocentriste " (la musique française est supérieure) tout en s'opposant aux nationalismes (qui menacent la musique en la divisant) et en prônant un internationalisme musical basé sur la raison. En affirmant que la musique française est un modèle de rationalisme, il la considère comme le candidat idéal pour montrer la voie vers ce rêve d'unité musicale entre les peuples.

Dans un texte de 1928, André Couroy dénonce la croyance selon laquelle la musique serait un langage international en soutenant que la musique, tout comme la langue, est intimement liée à la « race ». En prenant pour modèle Stravinsky, Cœuroy invite donc les compositeurs à rester fidèles à leur " race » en s'inspirant du folklore comme méthode de composition.

Dans la traduction française de leur brochure explicative rédigée en 1928, Arthur et Gertrude Moulton décrivent leur projet de Centre musical européen et appellent à l'union de l'humanité pour en assurer la réalisation. Leur vision utopique du cosmopolitisme rassemblant l'humanité autour d'un enjeu considéré comme universel - la sauvegarde de la musique - se présente comme une solution au matérialisme et à l'individualisme. Ultimement, le Centre permettra l'avènement de la paix dans le monde en développant la compréhension et la tolérance entre les peuples.

\section{NOTE SUR LES TRANSCRIPTIONS}

Dans la transcription des textes, nous adaptons tacitement la ponctuation, l'usage des majuscules, des italiques et des guillemets aux normes typographiques modernes. La graphie originale des noms est respectée. Toute autre intervention est signalée en note. Les paragraphes sont numérotés pour faciliter le repérage des passages cités ou glosés dans le commentaire qui précède la transcription des textes. Les coupures sont indiquées par «[...]» ou bien remplacées par un résumé de leur contenu présenté en italique et entre crochets. 


\section{L'universalisme francocentrique \\ « Les caractéristiques de la musique française " par Robert Bernard \\ (La Revue musicale, 1938)}

\section{COMMENTAIRE}

La mission de la France

Dans la première conférence du cycle L'universalité et le rayonnement de la musique française, prononcée à la Schola cantorum en février 1938 ${ }^{1}$, Robert Bernard ${ }^{2}$ expose une thèse où le nationalisme se déguise en universalisme : le langage musical français étant le plus universel, il peut contrer la montée des nationalismes. Bernard dénonce « la vague de nationalisme intransigeant et xénophobe qui sévit un peu partout en ce moment » (§3) et s'attache à démontrer le caractère universel de la musique française ${ }^{3}$, ce qui justifie sa mission antinationaliste et « civilisatrice " (à la manière impérialiste) : « propager notre patrimoine artistique à travers le monde » (§ 61) afin de «sauver » la musique en "redonn[ant] un élément de stabilité et de sécurité à l'édifice lézardé et chancelant de notre culture européenne » (§3).

Ces arguments avaient eu une certaine résonnance dans les revues musicales au lendemain de la Première Guerre mondiale, alors que la supériorité de la musique française sur les autres (et notamment l'allemande) est promue non seulement dans un esprit chauvin (qui est très présent), mais dans un but pacifiste, son caractère universel expliquant son rôle :

Notre musique sera d'autant plus universelle qu'elle sera plus française si rien n'était plus français que Racine ou l'art classique qui a ravi le monde pendant un siècle et demi. [...] La paix sera ce que notre labeur, notre sincérité, notre volontéle feront. [...] Les membres disparus de la Patrie attendent que les vivants achèvent leur victoire et que l'âme de la France libérée enchante et domine l'univers (Stoecklin 1919, p. 178).

\footnotetext{
1 Nous déduisons cette information du fait qu'un compte rendu de cette première conférence, par Arthur Hoérée, paraît dans le numéro de mars de La Revue musicale (Hoérée 1938). C'est ici même que le titre et le nombre (neuf) des conférences sont mentionnés.

2 La fiche biobibliographique sur Robert Bernard est disponible dans la section « Musicographes » du site Presse musicale: http://pressemusicale.oicrm.org/corpus/musicographes/ (consulté le 25 septembre 2017).

3 Bernard fait la distinction, au début de l'article, entre " langage musical français » et " musique française ", en spécifiant ne pas vouloir utiliser cette dernière expression qu'il juge moins " pure ». De la même façon que la langue existe avant la littérature, le langage musical français est un concept qui précède sa traduction personnelle en œuvres (la " musique française » considérée comme un corpus) de la part des différents compositeurs. Bernard n'approfondit pas davantage cette question pourtant très intéressante du point de vue esthétique (est-ce possible qu'un langage artistique existe avant les œuvres ?) et dans la suite de sa conférence utilise sans gêne «musique française » ou " esthétique musicale française " (voir à ce propos la note 12 ici même) pour « langage musical français ».
} 
L'universalité de la musique française

En quoi le langage musical français serait-il plus universel que les autres ? Bernard affirme que son caractère intellectuel, sa " soumission aux exigences de la raison » (§ 28) plutôt qu'à celles du sentiment - attitude qui se traduit dans les deux lois d'efficacité et de sociabilité -, enlève à la musique française les « aspects de pittoresque, de couleur » et " certains artifices techniques caractéristiques » qui identifient notamment la musique russe ou espagnole $(\S 32,33)$, mais aussi scandinave, tchèque, orientale ou anglaise (§ 48). Aucun «tempérament » ne se manifeste dans la musique française ( $(34)$, qui doit par conséquent « être considérée sur un plan d'universalité : elle s'accommode de tous les tempéraments et elle ne se reconnaît à aucun d'eux " (§33). En effet, guidé par une loi d'efficacité, le « désir primordial » du musicien français est l'adéquation entre la forme (technique musicale) et le contenu (\$22-24), « la conformité de la pensée et de son expression » ( $(38)$. (On remarquera, en lisant la conférence, que Bernard utilise le mot « expression » tantôt comme synonyme de technique - la voie par laquelle une idée est exprimée -, tantôt pour indiquer cette même idée qui doit être mise en forme.) Bref, la musique française décrite par Bernard résulte d'une attitude intellectuelle envers la composition qui, typique chez les Français (le conférencier n'explique pas pourquoi), lui permet de "véhiculer toutes les caractéristiques du style national d'un autre pays sans cesser d'être française » (§ 34).

Le résultat est une musique qui peine plus que d'autres à conquérir l'auditeur qui est généralement plus sensible aux effusions sentimentales qu'aux inventions de l'intellect $(\S 43,52)$. Bernard critique ici ouvertement la musique allemande qui « est pratiquement considérée comme la musique européenne " et qu'en raison de son sentimentalisme "trouve aisément des oreilles compréhensives, le sentiment étant, par excellence, la langue internationale » (§ 49). Le projet francocentrique de Bernard oppose, dans un élan d'autodéfense nationale mêlé de revanchisme antiallemand, de supplanter cette primauté universelle du sentiment par celle, moins aisée d'accès, de l'intellect. En se basant sur une loi de sociabilité qui ne se contente pas d'exprimer les individualités, mais qui " tient compte de toutes les exigences légitimes de l'auditeur» $(\S 58)$, la musique française serait donc un modèle de cohabitation respectueuse entre les peuples. Et « si la terre entière ne partage pas de telles vertus, tous les peuples civilisés peuvent et doivent les reconnaître et, du même coup, les admirer » (\$ 61) chez les Français, bien évidemment.

\section{Universalisme et nationalisme}

En quoi l'apologie de la musique française faite par Bernard diffère des positions nationalistes qu'il dénonce ${ }^{4}$ ? Il y a trois éléments qui les distinguent. Premièrement, Bernard oppose la civilisation (dont il parle) et la race. Selon lui, la " musique française » est le résultat d'une attitude culturelle des Français, plutôt que de leur génétique :

4 Une analyse d'articles de presse sur le concept de «musique française » fera l'objet d'une prochaine Note de terrain au sein de l'« Anthologie du PHEM ». 
«Ce n'est ni par des qualités proprement musicales, ni par des particularités de caractère que la musique française se distingue des autres musiques ; mais bien par une attitude intellectuelle, ou, si l'on veut, esthétique. Elle reflète une civilisation plutôt qu'une race » $(\S 53)$. Ceci a pour conséquence que, à la différence des positions nationalistes basées sur l'idée de " génie de la race ${ }^{5}$ " qui s'inscrit dans le sang, Bernard croit que les étrangers peuvent apprendre à faire de la musique française - c'est d'ailleurs son souhait, une universalité musicale bâtie sur le modèle français.

La troisième différence entre la pensée de Bernard et le nationalisme "génétique ", pourrait-on dire, est exposée dans un article que le musicographe publie en 1946, dans le premier numéro d'après-guerre de La Revue musicale - périodique qu'il dirige depuis le décès d'Henry Prunières en 1939. Bernard y affirme le caractère supranational des " génies » nationaux : s'il est vrai que, en raison d'une commune sensibilité, on ressent une grande familiarité avec la musique écrite par nos compatriotes, il est tout aussi vrai - Bernard se positionne ici contre celle qu'il appelle « la conception nationaliste de l'art » - qu'il y a des compositeurs qui contribuent à la musique de façon universelle, supranationale. Bernard prône donc pour une attitude " international[e], ou, mieux, extra-national[e], ou anational[e] » lorsqu'il s'agit "d'honorer, de propager, de défendre les œuvres des maîtres qui, à quelque pays qu'ils appartiennent, ont apporté à la civilisation un langage nouveau, une façon neuve de penser musicalement et de s'exprimer » (Bernard 1946, p. 47, italique de l'auteur).

La position de Bernard contraste avec ceux qui nient la possibilité qu'un " génie », complètement imbibé de sa " race ", reçoive des influences de la part de musiciens d'ailleurs. Cette idéologie puriste se trouve énoncée notamment dans un article de Georges Migot paru en 1924 dans la Revue Pleyel. «Il n'y a pas de nationalisme en Art, écrit Migot, mais il y a de la géographie et de l'ethnographie " (Migot 1924, p. 14-15). Migot demande qu'on arrête de trouver les influences de Wagner sur Debussy ou de Debussy sur Stravinsky, car un grand artiste ne l'est que « pour sa race, [...] par sa race et de sa race " (ibid., p. 15) : il invite donc, dans les « recherches de paternité pour un musicien français", à n'étudier que "les siècles de musique française » (ibid.). Les conclusions de Migot et de Bernard ne sont toutefois pas très divergentes : pour les deux, la musique n'est pas un langage international (un "volapuck ", écrit Migot à la p. $16^{6}$, un "espéranto ", disent d'autres) et le rôle d'un musicien français dans les échanges musicaux entre nations est d'être fermement « français ".

Nous reproduisons ci-dessous un long extrait de l'article écrit par Bernard en 1946, qui s'intègre bien à sa conférence de 1938 que nous transcrivons presque intégralement ci-après :

5 Concept tout de même employé par Bernard au $\S 63$, lorsqu'il évoque « ce pouvoir si particulier de nos disciplines techniques et esthétiques qui ne contrarient aucun génie racial étranger, mais les exaltent, les favorisent et les intensifient tous ».

6 La graphie correcte serait « volapük » (la langue internationale créée en 1879 ; l'espéranto suivra en 1887). 
Chaque peuple a non seulement le droit, mais le devoir de percevoir dans l'artiste de son sol les accents proprement nationaux de son message, ce en quoi sa sensibilité, son esthétique, son langage correspondent à l'esprit, à la civilisation et au goût de la nation à laquelle il appartient. D'un certain point de vue, Moussorgsky pour un Russe, Chopin pour un Polonais, Rameau pour un Français, Sibelius pour un Finlandais, Smetana pour un Tchèque, Falla pour un Espagnol, Rossini pour un Italien, Elgar pour un Anglais, Schubert pour un Autrichien, Bartok pour un Hongrois, etc. auront toujours un charme mystérieux, un pouvoir émotif qu'aucun étranger ne pourra pleinement saisir ni goûter. Cette vertu particulière peut nous toucher chez un artiste tout à fait secondaire sur le plan international tout autant que chez un génie universel, parfois même davantage. Tel est le fondement essentiel de la conception nationaliste de l'art. Mais il y a un autre point de vue qui ne contredit en aucune façon cette conception nationaliste, qui ne la diminue pas, ni ne l'altère, c'est celui où nous nous plaçons vis-à-vis des novateurs qui ont renouvelé la technique et les moyens d'expression de notre art. Sur ce plan, aucune considération nationale n'est légitime. [...]

Quand nous estimons que Strawinsky, Roussel, Falla ou Schoenberg doivent être connus de tous les mélomanes, ce n'est en aucune façon pour ce qu'il peut y avoir de spécifiquement russe, français, espagnol ou autrichien dans leur art, ce n'est même pas tant à cause de la valeur intrinsèque de leurs œuvres, mais parce qu'elles sont indispensables à l'intelligence de l'évolution du langage musical, parce que leur influence est universelle et que - qu'on le veuille ou non, qu'on les aime ou non, qu'on s'en réclame ou qu'on s'y oppose - nous ne pouvons pas faire abstraction de ce qu'ils ont apporté à toute l'humanité musicienne.

Nous n'ignorons pas que, pour nous, Debussy est tout autre chose que ce qu'il est pour un Argentin ou un Yougoslave [...]. Cependant, sous un certain axe, le message novateur et révolutionnaire de Debussy est exactement le même pour nous que pour un Argentin ou un Serbe. Et c'est pourquoi aucun peuple de la terre n'a le droit d'ignorer Debussy indépendamment de tout attrait ou de toute répulsion pour le génie français. De même, quand nous considérons l'atonalisme schoenbergien ou le dynamisme de Prokofieff, nous faisons abstraction de la sympathie ou de l'antipathie que nous pouvons avoir pour Vienne ou Moscou (Bernard 1946, p. 48-49). 


\section{TRANSCRIPTION}

[1] Répondant au vœu émis par l'Académie de Berlin en 17837 ${ }^{7}$, Antoine de] Rivarol écrivit en tête de son célèbre essai sur 1'Universalité de la langue française les lignes suivantes ${ }^{8}$ :

Une telle question, proposée sur la langue latine, aurait flatté l'orgueil des Romains, et l'histoire l'eût consacrée comme l'une de ses belles époques ; jamais en effet pareil hommage ne fut rendu à un peuple plus poli par une nation plus éclairée.

Le temps semble être venu de dire le monde français, comme autrefois le monde romain, et la philosophie, lasse de voir les hommes toujours divisés par les intérêts divers de la politique, se réjouit maintenant de les voir, d'un bout de la terre à l'autre, se former en République sous la domination d'une même langue. Spectacle digne d'elle que cet universel ${ }^{9}$ et paisible empire des lettres qui s'étend sur la vanité des peuples, et qui, plus durable et plus fort que l'empire des armes, s'accroît également des fruits de la paix et des ravages de la guerre!

[2] Rivarol parle ensuite de cette " honorable universalité de la langue française, si bien reconnue et si hautement avouée dans notre Europe ${ }^{10}{ }^{0}$... Hélas! à l'heure où l'on devrait pouvoir en dire autant de l'universalité de la langue musicale française, nous sommes bien obligés de reconnaître que, si d'innombrables preuves nous sont données de l'opinion que le monde civilisé se fait de notre art musical et que si ces preuves nous viennent de l'élite des nations étrangères, les assises de ce pacifique empire sont singulièrement mal assurées et que sa réalité n'est rien moins qu'évidente.

[3] La vague de nationalisme intransigeant et xénophobe qui sévit un peu partout en ce moment, sous divers aspects, rend à la fois inactuel et très actuel le problème de cette universalité. Inactuelle, car nous sommes plus loin que jamais de la voir reconnue des peuples étrangers, actuelle parce qu'elle constitue une des conquêtes qui, sur un point sans doute secondaire, permettrait de redonner un élément de stabilité et de sécurité à l'édifice lézardé et chancelant de notre culture européenne.

7 «Qu'est-ce qui a rendu la langue française universelle ? Pourquoi mérite-t-elle cette prérogative ? Est-il à présumer qu'elle la conserve? ». Sujet d'un concours proposé par l'Académie royale des sciences et belles-lettres de Berlin, dont le prix fut remporté, le 3 juin 1784, par Johann Christoph Schwab (1743-1821) avec sa Dissertation sur les causes de l'universalité de la langue françoise et la durée vraisemblable de son empire ([1803]2005) et par Antoine de Rivarol (1753-1801) avec son beaucoup plus synthétique discours De l'universalité de la langue francaise ([1784]1785). Huit textes ayant été soumis au jury du concours berlinois ont été recueillis en volume par Pierre Pénisson (1995). Le texte de Rivarol avait trouvé un certain succès éditorial dans l'entre-deux-guerres : une édition critique chez Delagrave en 1929 (par Marcel Hervier), la même année que celle annotée par Albert Chérel chez Hatier ; une autre chez Didier en 1930 (par Théodore Suran) ; une édition de la Société des bibliophiles havrais en 1934 ; et son inclusion dans les "Classiques Larousse » de 1936. Un nouvel engouement pour cette œuvre se registre à partir des années 2000 (disponible en audiolivre en 2003, elle fait l'objet de trois éditions entre 2009 et 2014).

$8 \quad$ Il s'agit des deux premiers paragraphes du Discours de Rivarol. Nous respectons la transcription de Bernard en ce qui concerne la graphie et la forme de mots. La deuxième phrase de la citation figurait en exergue à la première des huit conférences publiées par Bernard en 1930 au sujet des Tendances de la musique française moderne : "Situation de la musique française au XIX ${ }^{\mathrm{e}}$ siècle : les origines françaises et les influences étrangères » (Bernard 1930, p. 19). Cette conférence est par ailleurs très différente de celle transcrite ici.

9 Dans les éditions du Discours que nous avons pu consulter ([1784]1785; $1929 ; 1936 ; 1991$; Pénisson 1995, p. 129), le terme employé ici est " uniforme ». Une comparaison avec toutes les éditions disponibles à l'époque de Bernard permettrait de déterminer s'il s'agit d'une modification délibérée de sa part pour servir son propos universaliste, ou bien si l'adjectif « universel » est effectivement attesté.

10 Il s'agit du début du troisième paragraphe du Discours. 
$[4][\ldots]$

[5] Une vague de nationalisme spirituel sévit en France comme ailleurs, bien qu'elle soit très atténuée et surtout fort peu organisée. À tout prendre, elle n'est qu'une riposte à des provocations et elle ne vaut pas cher, car elle est basée sur la sauvegarde des intérêts matériels, bien plus que sur la juste notion d'une réalité envisagée objectivement ; c'est dans le camp des nationalistes les plus convaincus et les plus militants qu'on trouverait, sinon le plus, du moins les plus dangereux négateurs de l'universalité du langage musical français. [6] Je ne dis pas, à dessein, de la musique française, car le génie ou le talent des compositeurs, si indiscutables soient-ils, qui sont la parure de notre musique et l'une des preuves de la qualité souveraine de nos disciplines esthétiques et techniques, ne sont ou, du moins, ne pourraient être qu'une série d'accidents, d'heureux hasards, et, de toute façon, ils sont infiniment moins importants, au point de vue où nous nous plaçons, que ces disciplines considérées en elles-mêmes.

[7] Ce n'est pas tant la gloire des Montaigne, des Racine, des Pascal, des Molière ou des La Fontaine que Rivarol exaltait dans son essai, que la qualité universelle de la langue qu'ils parlaient. [...]

[8] A-t-on jamais envisagé notre musique sous cet angle et a-t-on mesuré la force des principes sur lesquels elle s'appuie et la souplesse des moyens d'expression qu'ils permettent, que dis-je, qu'ils suscitent et favorisent?

[9-17 : En faisant l'histoire de la musique française de l'époque baroque au début du XXe siècle, Bernard s'attache à démontrer qu'elle n'a pas pu atteindre dans le passé l'universalité qu'il défend pour différentes raisons d'ordre politique, culturel et pédagogique. Selon lui, c'est la méconnaissance de la musique française qui explique qu'elle ne soit pas déjà reconnue comme universelle ${ }^{11}$.]

[18] Il est malaisé de déterminer les caractéristiques de l'esthétique musicale française. Non pas, certes, que notre musique ne se différencie pas nettement de celles de toutes les autres nations ni que ses éléments constitutifs soient moins précieux que ceux de tout autre musique.

11 Lionel de La Laurencie (1920, p. 61), avance que la musique française baroque était internationaliste et propose un parcours entre les pièces intégrant les exotiques et les étrangers :

En ces temps troublés où l'Europe, disloquée, cherche à retrouver son équilibre, et où s'échafaude laborieusement la «Société des Nations ", il nous a paru de quelque intérêt de rechercher le rôle qu'a joué l'internationalisme dans l'ancienne musique française. C'est que, déjà, les musiciens contemporains d'Henri IV, s'associant indirectement aux généreuses pensées de l'auteur du « Grand Dessein », ménageaient le plus large accueil à la musique étrangère et l'art des sons devenait ainsi un lien international entre les peuples.

Fêtes royales et princières, divertissements et ballets de cour font appel à des éléments puisés au-delà des frontières. Tantôt ces spectacles se situent entièrement hors de France, voire chez les peuples les plus lointains, tantôt des scènes exotiques se glissent au sein de sujets mythologiques ou bouffons. Sans doute, l'importation étrangère apparaît alors destinée surtout à corser le spectacle par des costumes, des figurations et des danses extraordinaires, dont la singularité et la saveur constituent un puissant moyen d'attraction ; la musique qui accompagne les entrées de personnages exotiques ne réalise qu'un médiocre effort vers la couleur locale, car le folk-lore [sic] des peuples éloignés est encore à peine connu et de plus, on le tient en assez piètre estime. 
[19] Cette difficulté ne tient qu'à la qualité même de son essence.

[20] L'on prend assez communément un des effets de la discipline à laquelle obéit l'art français tout entier pour sa caractéristique la plus marquante, alors qu'il ne s'agit, à la vérité, que d'une apparence dont l'importance est très secondaire, pour peu qu'on ne la rattache pas à ses causes profondes, je veux parler de son agrément, fait d'élégance, d'esprit, de charme, de grâce et de légèreté.

[21] Il faudrait en finir, une fois pour toutes, avec ces étiquettes dont on affuble les divers génies nationaux et qui ne résistent pas à la moindre analyse. [...]

[22] Lorsqu'on parle de musique, on sous-entend un double phénomène : la technique musicale et l'expression, ou l'esthétique ${ }^{12}$.

[23] Cette dissociation est d'autant moins apparente que ces deux phénomènes sont étroitement liés et que la technique est plus étroitement adaptée à la pensée qu'elle revêt.

[24] Les musiciens français ont eu toujours le souci constant de l'expression - ou du style -, c'est-à-dire qu'ils n'ont jamais envisagé une pensée indépendamment de sa forme, ni une forme dissociée de la pensée qu'elle avait la mission d'exprimer. La corrélation entre le contenant et le contenu est sans cesse merveilleusement équilibrée chez nos grands maîtres, qu'ils se nomment Rameau, Couperin, Berlioz, Bizet, Fauré ou Debussy. Chez eux, la technique provient toujours de la pensée même - elle en dépend, et, par un phénomène de réversibilité qui est un des privilèges de l'art, la pensée est déterminée, pour une grande part, par les possibilités techniques dont dispose le créateur.

[25] Qu'il soit délicat, exquis, raffiné, un art qui a ce perpétuel souci de l'équilibre conceptuel, rien n'est plus certain. Jamais il ne vise de propos délibéré au grandiose, au sublime. Il y atteint, mieux qu'un autre, par sa perfection même, mais cela en se jouant, par hasard, par la grâce de la Providence, qui a donné du génie à tant de musiciens français. Viser au sublime, n'est-ce pas accepter un compromis vis-à-vis de soi-même, qui est indigne d'un esprit sain? Viser au grandiose, n'est-ce pas de gaieté de cœur et avec une troublante inconscience, vouloir plus qu'on ne peut? Ce n'est que l'excès de la sensibilité, l'éblouissement de la perfection, l'étincelle mystérieuse et non voulue venant magnifier l'humble labeur qui élèvent l'œuvre du probe artisan à la grandeur et à la profondeur.

[26] L'état de transe, de délire, l'ivresse sont des excitations morbides dont nos artistes se sont - fort heureusement - méfiés.

[27] Les musiciens français ont eu des préoccupations d'une tout autre nature. Ils ont tenté - avec quel succès ? - de dominer leur métier, de s'en rendre maîtres, et ils s'en sont remis au destin de leur insuffler la flamme du génie et de conférer à leur production la puissance persuasive et durable de la révélation et de l'inspiration.

[28] Intellectuelle, la musique française l'est par sa soumission aux exigences de la raison. Elle est sensible autant que ses représentants l'ont été, elle l'est d'autant plus qu'ils ne sont pas forcés de le paraître, mais que leur sensibilité sentimentale s'est inscrite, pour ainsi

12 Remarquons la signification du mot « esthétique » chez Bernard ( $(6,18,22,32,36,53,63)$. Ici, il est utilisé en couple avec le mot " expression ", dans une acception antinomique par rapport à « technique ". L'esthétique est donc ce qui précède la technique - l'idée, l'esprit, le contenu de la musique, son « attitude intellectuelle » (§53). Cette acception du terme est confirmée aux $\S 6$ et 63, où Bernard oppose les "disciplines esthétiques et techniques ", et au $\S 36$, où il parle de "l'unité de l'esthétique musicale qui a présidé à toute notre musique » (c'est-à-dire, indépendamment des différences techniques et stylistiques entre les différents compositeurs français). L' ' esthétique musicale française » qui fait l'objet de la conférence est donc définissable comme une « attitude intellectuelle » qui peut prendre de différentes formes selon la technique musicale qui la traduit. Voir aussi notre commentaire ci-dessus, note 3. 
dire, malgré eux, dans l'inaltérable substance qu'ils ont créée. Quand on loue la pudeur sentimentale des artistes français, on touche à l'une des caractéristiques essentielles de leur production, car cette pudeur provient de multiples qualités qui sont primordiales chez eux : le tact, la mesure, la conscience, le goût du vrai, du simple, du naturel. Non seulement ils se sont abstenus d'enfler leur voix, de prétendre à une grandeur qu'ils étaient incapables d'atteindre, mais encore ils ont évité la confidence pathétique qui n'est réellement touchante que lorsqu'elle est involontaire, qu'elle jaillit directement du cour et qu'elle apparaît, mystérieuse et imprévue, avec cette grâce infinie d'un sourire d'enfant, d'un premier amour ou d'une eau transparente, toutes choses qui sont sans apprêt, dont la poésie profonde émane d'éléments naturels et inconscients.

[29] Il y a de beaux désespoirs simulés, il y a d'émouvantes passions dont les artifices sont théâtraux ; mais de quels prix sont les plus fastueux simulacres comparés à un sentiment craintivement naïf et spontané.

[30] Il ne s'agit pas ici d'inconscience, mais de tact et de réserve, en un mot, de pudeur. $[\ldots]$

[31] Pour un Beethoven, dont le génie a été fulgurant et qui n'est pas responsable de toute la littérature qu'on a faite sur lui, que d'épaves échouées lamentablement dans cette course au sublime, dans ce combat dérisoire avec des forces hostiles !

[32] La réserve sentimentale qui préside à l'esthétique musicale française contribue, pour une large part, à la connaissance de cette esthétique qui, par ailleurs, est d'accès difficile dans la plupart des pays étrangers où l'on est habitué à apprécier la personnalité d'une musique sous ses aspects de pittoresque, de couleur, ou par certains artifices techniques caractéristiques.

[33] Il n'y a rien, dans la musique française, quel'on puisse opposer au pittoresque rythmique de la musique espagnole ou russe et, sur un plan plus intellectuel, à leur caractère racial. La musique française doit être considérée sur un plan d'universalité ; elle s'accommode de tous les tempéraments et elle ne se reconnaît à aucun d'eux. [...]

[34] [...] Il faut chercher à délimiter [les] apparentes antinomies [entre les différents auteurs] en délimitant la véritable nature de l'unité musicale française. Il faut tout d'abord [...] écarter toute notion de tempérament. La nostalgie slave, par exemple, avec son charme douloureux et dont le pittoresque est rehaussé par des influences orientales, n'a pas d'équivalence dans la conformation de la musique française. Universelle par son essence, celle-ci peut véhiculer toutes les caractéristiques du style national d'un autre pays sans cesser d'être française.

$[35][\ldots]$

[36] Où faut-il chercher l'unité de l'esthétique musicale qui a présidé à toute notre musique? [...]

[37] En tout premier lieu - et c'est là le nœud de la question qu'il convient d'expliquer, de délimiter et aussi de développer -, notons une loi essentielle, génératrice : la loi d'efficacité. [38] Le musicien français à un désir primordial, celui du style, du style au sens littéraire du mot, c'est-à-dire de la conformité de la pensée et de son expression. Ailleurs, on se soucie tour à tour de rehausser la pensée par l'éclat de l'expression ou de se faire pardonner la médiocrité de l'expression par la profondeur, la hauteur de la pensée. Selon son tempérament, on prétend atteindre la pensée dans son intégrité, indépendamment des moyens qui servent à l'exprimer, ou l'on prétend se satisfaire du jeu mathématique des agencements sonores, sans s'attarder à leur valeur psychologique. À un autre point de vue, et sans pousser les choses à leur extrême conséquence, on marque une indifférence évidente pour l'opportunité, pour l'efficacité des formes et du langage, et l'on cherche à inscrire une pensée dans une forme ou un langage qui ne sont 
pas déterminés par cette pensée même. Les plus grands génies d'Outre-Rhin ont, plus ou moins, pâti de cette erreur conceptuelle. J. S. Bach lui-même n'y a pas toujours échappé en inscrivant les pensées les plus diverses dans un langage manifestement moins divers. Je cite son nom à dessein, car, de tous les musiciens de tous pays et de tous temps [sic], il est le plus universel et le plus équilibré. Le déséquilibre entre la forme et le fond est infiniment plus fréquent et plus regrettable chez tous les grands classiques $d u \mathrm{XVIII}^{\mathrm{e}}$ siècle allemand, même chez Beethoven, qui eut pourtant, au suprême degré, le génie de forcer sa technique à des fins nouvelles. Avec Reger, Brahms, Bruckner, Mahler, Strauss, Busoni, Schoenberg, Schreker et quelques autres, nous rencontrons de pires conséquences à ce déséquilibre. En France, il n'a jamais sévi qu'à la suite d'influences manifestement étrangères. Nos grands musiciens ont toujours su s'en préserver, et ils n'ont pas écrit une note qui ne soit d'une entière efficacité, car leur musique dépend étroitement de la pensée qu'elle veut exprimer, et, réciproquement, cette pensée ne saurait être traduite par d'autres moyens d'expression que ceux dont dispose le musicien.

[39] Logique, ordonnée, la musique française est intellectuelle par sa discipline, par les contraintes extérieures et intérieures qu'elle se prescrit et elle vaut principalement par l'art avec lequel elle s'y soumet.

[40] Cette efficacité de la musique française est proprement musicale ; c'est ce qui la rend si difficile d'accès. Quand le musicien se propose d'émouvoir et que l'efficacité de son œuvre tient à ce que celle-ci atteint son but, tout le monde en convient et le loue. Mais, quand cette efficacité porte sur la substance musicale elle-même, seuls les initiés en sont touchés.

[41] [Bernard fait ici une métaphore avec l'architecture. En comparant la construction d'un édifice avec la composition musicale, il montre que si les critères de jugement sont des qualités extérieures (Bernard dit "ampleur de dimension "), l'architecte (ou en l'occurrence, le compositeur) rencontrera l'appui immédiat du public. En revanche, une cuvre construite d'après des contraintes spécifiquement architecturales (lire ici "compositionnelles ") ne sera appréciée que d'un public éduqué, connaissant les «données du problème»].

[42] À génie égal, il est plus facile pour un étranger de prétendre au sublime (au sens usuel, banal du mot) que d'être simplement honorable en France. C'est en France que l'exercice de la musique rencontre les plus grandes difficultés pour le créateur [...]. L'inspiration ne rachète rien, le métier n'y a guère de prix. [...] Les vertus primordiales de l'art français portent sur la discipline de l'intelligence et de la sensibilité bien plutôt que sur leur mode d'extériorisation. Reflétant une culture plutôt qu'une forme de sensibilité, il doit non seulement être le reflet de cette culture, mais encore sa parure : il doit la refléter avec éclat, avec les qualités les plus rares et les plus précieuses.

[43] La sensibilité réceptive, quelque vive qu'elle soit, n'est vulnérable, en France, que pour autant qu'on aille la conquérir dans sa forteresse : l'esprit. Le Français ne veut se livrer à son émotion que si la perfection du style, la maîtrise de la technique, la sûreté du goût ont satisfait pleinement son sens critique toujours en éveil. Une œuvre musicale authentiquement française ne force pas l'admiration, moins encore une adhésion émotive ; sévère pour elle-même, difficile dans le choix de ses moyens, elle doit être écoutée avec la plus grande lucidité critique. [...] Rien n'y est laissé au hasard, et sur des plans sans doute très divers et inégaux, toutes les œuvres de nos grands musiciens sont exemptes de ces pages vides de pensée - ou de musique - qui diminuent la valeur intrinsèque de la plupart des grandes œuvres étrangères. [...] La subtilité des modulations, la pureté des contours mélodiques, la logique des agencements harmoniques, l'ingéniosité de la polyphonie et de l'instrumentation y sont des vertus constantes et sans cesse agissantes. L'invention, dans quelque domaine que ce soit, n'y tarit jamais, et l'on a la certitude, en les abordant, 
que rien n'y est laissé au hasard ou à la facilité, pas plus qu'au procédé de développement conventionnel.

[44] [L'exemple de Ravel.]

Nulle part ailleurs, on a mieux compris ce que c'est que la vérité, en matière de musique, et que la musique ment quand ellen'invente pas, qu'elle perd sa dignité dès qu'elle exploite des formules, qu'elle est avant tout et par-dessus tout poésie, c'est-à-dire découverte, invention ${ }^{13}$. [L'exemple de Debussy dans les mots d'André Suarès.]

$[45,46][\ldots]$

[47 : Méconnaissance de la musique française de la part des étrangers.]

[48] [...] Comme nous l'avons vu, c'est à des éléments de pittoresque qu'on reconnaît ordinairement les divers styles musicaux. Le mélomane le moins cultivé distingue la musique russe de l'espagnole, la scandinave de l'orientale, et même la tchèque de l'anglaise. Distinctes par leurs rythmes, par leurs harmonies, par la courbe de leur mélodie, ces musiques sont encore reconnaissables à leur caractère expressif qui traduit plus ou moins clairement le génie psychologique de leurs nations respectives.

[49] La musique allemande, grâce à l'envergure et à la popularité des musiciens qui l'ont illustrée, est pratiquement considérée comme la musique européenne, et, comme ses sources d'inspiration ${ }^{14}$ autant que ses tendances sont d'ordre sentimental, elle trouve aisément des oreilles compréhensives, le sentiment étant, par excellence, la langue internationale.

[50] L'universalité de l'école française, aussi indéniable que celle de l'école allemande, si l'on juge des choses in abstracto, est un fait beaucoup moins évident. Un art qui est sous le signe de l'esprit, qui tend et atteint à la vérité psychologique et dont les vertus sont avant tout d'ordre intellectuel, ne saurait ${ }^{15}$ rallier d'unanimes suffrages, car la raison n'est pas, à proprement parler, un phénomène universel. Je veux dire par là que la conduite d'un raisonnement diffère assez considérablement d'un peuple à l'autre et, ce qui importe davantage, que toutes les nations ne se soucient pas que l'œuvre d'art satisfasse en tout premier lieu leur intelligence.

[51] L'art français (cette considération ne s'applique pas uniquement à la musique) s'adresse toujours à l'esprit et, en France, l'esprit contrôle toujours les élans de la sensibilité en les disciplinant.

[52] Toutes les caractéristiques de la musique française proviennent de ce postulat qui ne nous lassons pas de le répéter - ne restreint en aucune façon la valeur émotive de notre musique. Tout au plus, la rend-elle plus distante et d'un accès moins aisé.

[53] Ce n'est ni par des qualités proprement musicales, ni par des particularités de caractère que la musique française se distingue des autres musiques ; mais bien par une attitude intellectuelle, ou, si l'on veut, esthétique. Elle reflète une civilisation plutôt qu'une race. Capable de véhiculer tous les sentiments, [...] [e]lle tend constamment vers la simplicité, vers la vérité psychologique, vers la perfection de la forme. Toujours claire, directe, elle prétend à l'équilibre, tandis que la plupart des autres musiques recherche l'exceptionnel et l'excessif. Sa force - et aussi sa faiblesse si on considère les choses à un point de vue que

13 Bernard choisit ici donc le terme " poésie » dans son acception étymologique (de poiésis : création, fabrication).

14 Bernard écrit « d'inspirations ».

15 Bernard écrit « sauraient». 
je qualifierai de "pratique »-c'est de ne jamais élever la voix plus qu'il n'est nécessaire, d'éviter tous les procédés qui ne sont pas entièrement légitimes, qui violentent le bon goût, qui rompent l'harmonie. Renonçant au suffrage du "vulgaire ", nos grands musiciens sacrifient toujours la quantité à la qualité, ils préfèrent être incompris que d'user des mensonges conventionnels qui frappent l'imagination de l'auditeur. Avec tact et mesure, ils disent en une langue châtiée et dont la plus précieuse vertu est la parfaite adéquation du fond et de la forme, ils disent ce qu'ils ont à dire [...]. Ils savent que la pudeur sentimentale n'est pas une vaine coquetterie, mais le signe de la véritable émotion ; l'emphase et la grandiloquence sont bannies de leur style qui est toute transparence, toute pureté, toute délicatesse ; ils usent même rarement et avec la plus grande circonspection de l'éloquence. [54] [...]

[55] «Il ne s'agit pas tant de frapper fort que de frapper juste ». Telle est la loi immuable que Lucien Dubech ${ }^{16}$ a formulée pour l'art français. [...] Nos auteurs n'ont jamais voulu composer avec ce qui constitue les vices du public réel.

$[56,57][\ldots]$

[58] Ils demeurent un exemple pour tous les peuples en ce sens que leur dédain du succès basé sur les compromis avec leur idéal a constamment été contrebalancé par un ensemble de lois artistiques qui tiennent compte de toutes les exigences légitimes de l'auditeur. Ces lois sont celles de la politesse, du goût et de la mesure - ce sont des lois de sociabilité. [...] [59] Les plus grandes audaces techniques qui, loin de les effrayer, les ont toujours, au contraire, attirés comme le plus sûr moyen de donner une forme vivante, neuve, incisive à leur message $[\ldots]$.

[60] [...] N'est-ce pas là, encore, une vertu de politesse et d'éducation : ce tact qui, dans la vie sociale, permet de dire sa pensée sans être blessant, de garder son indépendance et d'être fidèle à ses convictions sans être hargneux ou incivil, d'exposer des idées profondes et savantes sans ennuyer ni humilier des partenaires ignorants.

[61] Si la terre entière ne partage pas de telles vertus, tous les peuples civilisés peuvent et doivent les reconnaître et, du même coup, les admirer. Notre premier devoir est d'en prendre conscience afin de propager notre patrimoine artistique à travers le monde.

$[62][\ldots]$

[63] [...] Le témoignage des étrangers les plus autorisés, à travers les siècles, et, très particulièrement, cette impulsion génératrice que notre musique n'a cessé de donner aux maîtres étrangers qui, par quelque côté - parfois plusieurs - ont subi l'influence de notre musique, nous a paru l'un des arguments les plus convaincants, parallèlement à cette force attractive de Paris vis-à-vis des meilleurs musiciens de tous les temps et de tous les pays, de même que ce pouvoir si particulier de nos disciplines techniques et esthétiques qui ne

16 Lucien Dubech (1881-1940), critique littéraire, théâtral et sportif (L'Action française, Candide), militant nationaliste et royaliste. La phrase que Bernard attribue à Dubech est une variante sur une maxime utilisée de façon variable chez différents auteurs. Dans sa tragédie Wallstein (1809), Benjamin Constant (1767-1830) écrivait que « c'est en France qu'a été inventée cette maxime, qu'il valait mieux frapper fort que juste. Contre un pareil principe il faut des règles fixes, qui empêchent les écrivains de frapper tellement fort qu'ils ne frappent plus juste du tout » ( $\$$ "Réflexions sur Wallstein », p. xxvi). Chez Honoré de Balzac (1799-1850), on la retrouve dans le sens inversé adopté par Dubech - mais appliquée aux conflits conjugaux : «La puissance ne consiste pas à frapper fort ou souvent, mais à frapper juste » (Balzac [1830]1838, « Méditation V. Des prédestinés », § XVII du « Catéchisme conjugal »). 
contrarient aucun génie racial étranger, mais les exaltent, les favorisent et les intensifient tous - les plus convaincants, disais-je, pour étayer notre thèse et vous rallier à ses postulats et à ses conclusions.

\section{L'illusion internationaliste "Sous le signe du national " par André Cœuroy \\ (Le Ménestrel, 1928) ${ }^{17}$}

\section{COMMENTAIRE}

\section{Le côté irréductiblement national de la musique}

$\mathrm{Si}$, dans la conception universaliste francocentrique exprimée par Bernard, une musique nationale (en l'occurrence, la française) peut servir de modèle pour les compositeurs de tout pays, André Cœuroy ${ }^{18}$ - dix ans avant la conférence de Bernard et en précédent celui-ci dans le poste de rédacteur en chef de La Revue musicale croit au contraire que toute forme d'internationalisme musical n'est qu'une illusion. Plus particulièrement, Cœuroy nie l'universalité du langage musical de la tradition tonale occidentale : ce n'est pas parce que les styles allemands et italiens ont été exportés partout en Europe qu'il faut en "conclure à l'internationalisme des sons » (§1). Cette illusion est pourtant très répandue - "Quoi ? Est-ce que la musique de Beethoven n'est pas internationale ? ", semble s'exclamer un lecteur étonné (§ 2). Ce à quoi Cœuroy répond sur un ton élitiste : 1'« auditeur moyen " ne fait pas la différence entre, d'une part, la surface des phrases et des gestes musicaux (ce qui fait appel au côté le plus " animal " de sa sensibilité - le paladin, l'amoureux, le porc qui sont en lui [ibid.]) et, d'autre part, le « quelque chose d'irréductible » qui est intimement lié à la " race » dont chaque compositeur est issu. " Ainsi donc », comme le résume Philippe Gumplowicz, "ce qui ne serait pas "national" dans la musique serait banal ", tandis que "l'irréductible, le singulier - le génie, par conséquent - se placent "sous le signe du national" " (Gumplowicz 2004, p. 110). Pour Cœuroy - qui se sert d'une longue citation de Lionel Landry pour énoncer son propos -, la langue musicale est comme la langue parlée, et donc le côté « irréductiblement » national de la musique mène à une distance également irréductible entre les peuples, voués à l'incompréhension (musicale) réciproque. S'il est possible d'apprendre plusieurs langues, on aura toujours une seule langue maternelle - l'expérience de traducteur de Couroy a probablement eu une influence sur cette conception qu'il étend à la musique.

17 Le texte repris dans Le Ménestrel est l'introduction au Panorama de la musique contemporaine que Couroy venait de publier aux éditions Kra et dont 13 éditions paraîtront entre 1928 et 1930 (Cœuroy [1928a]1930).

18 La fiche biobibliographique sur André Cœuroy (nom de plume de Jean Belime) est disponible dans la section «Musicographes » du site Presse musicale : http://pressemusicale.oicrm.org/corpus/musicographes/ (consulté le 25 septembre 2017). 
Contre le modernisme musical cosmopolite

La cible de Couroy, on le comprend au $\S 4$, c'est la musique moderne. La constitution d'une langue musicale qui s'émancipe des traditions nationales et qui peut être utilisée par les compositeurs de toute provenance préoccupe un grand nombre de musicographes de cette époque "éprise d'internationalisme » (§ 4), où des institutions comme la Société internationale de musique contemporaine (SIMC) proposent l'idéal (en effet, le " mirage ", selon Cœuroy) d'une " musique moderne mondiale » (Dent 1923, p. 59) où des moyens techniques communs l'emportent sur les différences de $"$ race ${ }^{19}$. (Remarquons toutefois que la crainte d'une « influence de l'époque, unifiant toujours et partout les âmes et les modes " avait déjà été exprimée par Raymond Bouyer en 1905, et que sa conclusion allait dans le même sens que le fera Cœuroy : "Mais, sous le costume imposé par l'époque, comme la race persiste, comme la chair demeure sous les parures de l'esprit! » (Bouyer 1905, p. 35)). Cinq ans après la publication, dans les pages de La Revue musicale, du manifeste de la simc écrit par son président Edward J. Dent ${ }^{20}$, Couroy dresse donc un bilan de ce « mirage de fraternité sonore ", dont il constate la faillite : "À l'usage, il apparut que la création d'un esprit et d'un langage musical européen était chose impossible » $(\S 4)$; des festivals annuels de la SIMC, " chacun revient avec le sentiment aigu qu'il appartient à un groupe national, que ce groupe, s'il n'est pas meilleur que les autres, est au moins fort différent » (ibid.).

La relation entre modernité, internationalisme et chaos fait l'objet d'un article de 1939 par Joseph Estève. En comparant son époque à l'hellénisme - lorsque les conquêtes d'Alexandre le Grand ont créé une civilisation commune réunissant l'Occident et l'Orient -, il constate que l'internationalisme donne lieu à une "production abondante et hâtive, recherche du nouveau qui semble s'inspirer le plus souvent du désir d'étonner ou d'éblouir » (Estève 1939, p. 171) :

Les tendances qui se firent jour dans la sculpture hellénique au lendemain de la mort d'Alexandre, se manifestent de nos jours, exactement les mêmes, mais avec plus d'ampleur, la terre étant devenue plus petite, les peuples qui se mêlent plus nombreux, plus grande et plus indiscrète leur ardeur à s'introduire les uns chez les autres pour leur plus grand dommage. [...] Lorsque de tous les points de l'horizon se donnent pour ainsi dire rendez-vous les formes d'art les plus différentes, les conceptions les plus contradictoires, il en résulte un chaos où il est bien difficile de se reconnaître. [...]

[L]es conséquences d'un internationalisme qui tend à ne plus connaître de limites [...] sont fâcheuses pour l'art, dont elles contrarient, elles retardent le progrès (ibid., p. 175-176).

19 Sur le manifeste de la SIMc et l'opinion des critiques français sur cette société, voir Lazzaro [à paraître], chap. 7 .

20 La SIMc a été fondée en 1922. Son premier festival a eu lieu à Salzbourg, du 2 au 7 août 1923.

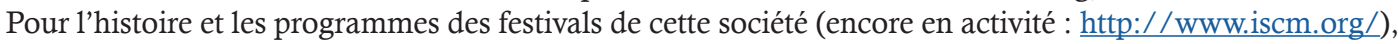
voir Haefeli 1982. 


\section{Cosmopolitisme et internationalisme}

La crainte d'un modernisme musical cosmopolite où les frontières seraient nivelées (Vuillemin 1923) au lieu d'un sain échange entre les nations qui gardent leur indépendance et leur identité (l'inter-nationalisme) ressort fréquemment de la plume des musicographes français des années 1920 : Roland-Manuel s'exprime en faveur de cet « internationalisme intelligent " (Roland-Manuel 1928, p. 390) ou " clairvoyant » (Roland-Manuel 1925, p. 9) ; Georges Migot valorise les rencontres internationales en tant qu'occasions de connaissance réciproque - mais pourvu que la "satisfaction artistique " que cette connaissance procure ne donne pas lieu à des emprunts ou à des échanges (Migot 1928, p. 505 ${ }^{21}$ ). Le fait que l'internationalisme soit conçu par les musicographes non comme une abolition de l'entité nation, mais comme une coopération entre nations (le " concert européen " que certains musicographes regardent avec sarcasme ${ }^{22}$ ) est un réflexe de "l'imaginaire internationaliste » libéral qui, à partir de la seconde moitié du XIX ${ }^{e}$ siècle, a inspiré la création d'organisations telles que la Croix-Rouge internationale, l'Union postale internationale, l'Union télégraphique internationale, jusqu'à la Société des Nations en 1919 (voir Iriye 2000, 2002 ; Sluga 2013).

$\mathrm{Au}$ lendemain de l'institution de la Société des Nations et avant celle de la SIMC, Henri Collet et Jean-Aubry imaginent, indépendamment l'un de l'autre, des sociétés musicales inter-nationales (une "Société internationale de musique " pour Collet, une "Société inter-nationale de musique " pour Jean-Aubry) vouées au dépassement du " nationalisme ombrageux " (Jean-Aubry 1922, p. 244) mais basées sur le principe que "le mot "internationalisme" (inter-nationes) ne signifie pas autre chose qu'une entente entre les nations" (Collet 1919, p. 307).

Couroy constate que, dans les faits, ces rencontres, loin de favoriser le respect dérivant de la connaissance réciproque ont durci encore plus les positions chauvines : "Chaque nation prétend se tailler sa part dans le butin, prélever son lot, et, sur cette matière conquise, imprimer sa griffe en la faisant la sienne $(§ 5)^{23}$. La comparaison ne fait qu'accentuer les identités : "Vagues jusqu'ici et flottantes, les frontières musicales se dessinent avec fermeté. [...] Chaque musique nationale prend conscience de son unité, retrouve le sentiment de la tradition » (ibid.).

Quelques exceptions à ce discours dénonçant l'internationalisme comme une illusion ou carrément une menace existent, bien qu'elles soient assez marginales et exprimées, le plus souvent, par des non-Français. Le compositeur grec Manolis

\footnotetext{
21 Dans la même enquête de la revue Musique où on trouve la réponse de Migot, Aloys Fornerod exprime des positions encore plus racistes et xénophobes contre l'« esthétique internationale» des «Barbares de génie » (Fornerod 1928, p. 585). En France, à partir de la guerre de 1870, le mot «barbare » était souvent utilisé pour désigner les Allemands (Joye 2016, p. 105).

22 Pour un billet humoristique au sujet du « concert musical européen », voir Margat 1935.

23 Il est intéressant, à ce propos, de lire les comptes rendus des festivals simc écrits par Henri Gil-Marchex (1923 ; 1924), dont la préoccupation principale est de voir si la France est bien représentée (numériquement et qualitativement) et sort donc gagnante de la comparaison.
} 
Kalomiris (1883-1962), par exemple, répond à une "Consultation sur la musique contemporaine " proposée en 1924 par Le Courrier musical que, selon lui, la musique contemporaine se dirige vers un «langage musical moderne enrichi de trésors musicaux de toutes les nations " (Kalomiris 1924). Les rencontres internationales seraient donc une véritable occasion d'échanges entre des identités très prononcées, mais qui gagnent à se "métisser " en apportant chacune ses forces distinctives en vue d'un langage commun. Le critique belge Arthur Hoérée écrit en 1928 que sa « situation ethnique à l'intersection de deux civilisations [1]'a habitué, dès l'enfance, à subir profondément et sans heurts deux cultures réputées incompatibles » et qu'il est donc optimiste quant à «l'interpénétration musicale des races » (Hoérée 1928, p. 216).

Où se trouve le "national»?

La dernière partie du texte de Cœuroy est consacrée à considérer comment rendre une musique nationale, et plus particulièrement, où les nouvelles nations nées avec le Traité de Versailles (comportant la dissolution de 1'Empire austro-hongrois) peuvent puiser pour se donner une identité nationale assez forte leur permettant de s'affirmer comme des pairs sur l'échiquier musical international. Qu'on le veuille ou non, on ne peut pas se soustraire au caractère " global » - dirait-on aujourd'hui - du monde contemporain.

Cœuroy met en garde contre la tentation de s'approprier le langage des "génies » dont l'influence dépasse les frontières : Debussy, Schoenberg, Stravinsky. Couroy ne condamne pas ici l'internationalisation des « génies » en raison d'une prétendue pureté « raciale » de leur art, mais en tant qu'obstacle à une construction identitaire ancrée de manière génuine dans la " source indigène $[. .$.$] de chaque pays » (§ 6). Cette source est$ le folklore local, qui doit inspirer les compositeurs non seulement en tant que modèle - « la curiosité de l'ethnologue serait satisfaite, mais l'art serait mince » (§9)-, mais en tant que méthode, tel qu'enseigné par Stravinsky $(\S 10)^{24}$.

\section{TRANSCRIPTION}

[1] Longtemps l'on a cru que la musique était un langage international. Mais chaque race a son style musical. Parce que, durant des siècles, ce fut la langue musicale allemande ou la langue musicale italienne que parlèrent les compositeurs du monde entier, on en voulait conclure à l'internationalisme des sons.

[2] Quoi ? Est-ce que la musique de Beethoven n'est pas internationale ? Ou, sur un autre plan, celle de La veuve joyeuse ${ }^{25}$ ? L'illusion vient de ce que l'auditeur moyen n'entend pas le langage réel de la musique. Ce qu'il entend, c'est ce qui s'adresse au paladin, à l'amoureux ou, sauf respect, au porc, qui sont en lui. Ce qu'il y trouve d'international, ce sont les images évoquées, qui a leur tour suggèrent des tours de phrase universels ; et ces tours sont

24 C'est en effet sur cette « leçon de Stravinski » que Cœuroy ouvre le premier chapitre de son livre, "À la recherche d'un style».

25 Die lustige Witwe (1905), opérette de Franz Léhar sur un livret de Victor Léon et Leo Stein, est effectivement considérée comme un succès international qui dépasse ses origines viennoises. 
beaucoup moins des phrases musicales que des gestes sonores aussi aisés à comprendre que la main sur le cœur ou le clignement d'œil égrillard. En toute musique, il y a quelque chose d'irréductible. Un subtil esthéticien de ce temps, Lionel Landry, dans sa Sensibilité musicale, a montré combien précaire était ce privilège de «langue internationale » communément attribué à la musique :

Le problème de la compréhension internationale de la musique n'est pas essentiellement différent de celui de la compréhension d'une langue parlée.

Chacun a éprouvé, en traduisant, qu'il est, dans une même littérature, des auteurs qui se prêtent ou se refusent à la traduction, non seulement pour des raisons de vocabulaire ou de syntaxe, mais surtout au fond, parce que la pensée informulée qui engendrait leur discours est en harmonie ou en discordance avec la nôtre. De même qu'il est des frontières particulières pour les vocabulaires d'autres pour les syntaxes, il en est pour les divers dialectes de la langue informulée intérieure, et il est possible que ces dernières coïncident partiellement avec celles des sensibilités musicales ${ }^{26}$.

[3] Un Fauré, musicien pur et ne parlant que la langue musicale de son pays, ne sera jamais compris à l'étranger. Brahms ou Bruckner ne seront jamais compris en France comme les comprennent leurs compatriotes. La musique orientale n'est pas comprise des Européens. De la musique nègre, nous ne percevons vraiment que des signes tout extérieurs.

[4] Ces vérités commençaient d'être perçues quand, après la guerre, fut fondée la Société internationale de musique contemporaine (SIMC). Les musiciens rêvaient d'États-Unis d'Europe. C'était un mirage de fraternité sonore. À l'usage, il apparut que la création d'un esprit et d'un langage musical européens ${ }^{27}$ était chose impossible. De ces rencontres, chacun revient avec le sentiment aigu qu'il appartient à un groupe national, que ce groupe, s'il n'est pas meilleur que les autres, est au moins fort différent, qu'il s'en distingue par des traits précis dont un secret instinct commande d'accentuer encore la vigueur. Jamais époque ne fut plus éprise d'internationalisme, et jamais les compositeurs qui marquent n'ont été plus nettement nationaux. Jadis les historiens de la musique cultivaient leur jardin aux trois allées bien alignées : l'allée italienne, l'allée allemande et l'allée française. Tout le monde passait par l'une des trois. C'était bien commode pour les gardiens du lieu ; bien commode aussi pour les promeneurs. Aujourd'hui, il n'est pas de petit peuple qui ne veuille sa grande route. Il se peut qu'il l'empierre avec du gravier dérobé à l'une des trois allées antiques (ou même à toutes les trois ensembles). Mais, pourvu qu'il y marche en sifflotant un petit refrain populaire, il a sa route nationale dont il est fier.

[5] Ainsi se précisent, pour la cartographie des sons, les nationalités musicales. Vagues jusqu'ici et flottantes, les frontières musicales se dessinent avec fermeté. Il semble même parfois qu'elles vont se dresser comme des barrières. Le plus distrait des auditeurs

26 L'auteur cite ici Landry 1927, p. 145. Lionel Landry (1875-1935) est l'auteur de plusieurs articles à sujet esthétique dans la presse musicale, qu'il est possible de consulter dans la banque de données du PHEM à partir de cette page de recherche : http://1mhsbd.oicrm.org/list/?csrfmiddlewaretoken=bszfavWqGM8PUNsjO9k16DzsxO4PFUhz\&auteur nom $=$ landry, + lionel\&titre $=\&$ date $=\&$ mot cle nom $=\& p d f$ text=\&source=\&tousIndex calcul=\&projet nom=\&type=\&submithttp://1mhsbd.oicrm.or $\quad$ (consulté le 25 septembre 2017).

27 Couroy écrit « européen ». 
soupçonne une division toute nouvelle de la matière musicale. Avec l'éveil de l'âme aux accents des chansons populaires retrouvées, rééditées, réapprises, voici qu'une sorte de partage s'accomplit. Chaque nation prétend se tailler sa part du butin, prélever son lot, et, sur cette matière conquise, imprimer sa griffe en la faisant sienne. Chaque musique nationale prend conscience de son unité, retrouve le sentiment de la tradition, affirme l'existence d'une chaîne ininterrompue d'œuvres dans le passé et dans l'avenir, entre lesquelles peut et doit s'établir le lien d'une inspiration commune. Sans avoir lu Fontenelle, musiciens et auditeurs se rendent compte que les différentes idées musicales sont " comme des plantes et des fleurs qui ne viennent pas également en toute sorte de climat $^{28}$ ". Si détestable soit le nationalisme en esthétique, c'est là le fait essentiel de la musique contemporaine.

[6] Cette conscience musicale personnelle que chaque peuple cherche présentement à acquérir est sans cesse menacée par les fantômes enveloppants que créent les génies individuels. Le debussysme a presque disparu en France, mais il est en pleine action à l'étranger. Le schœnbergisme envoûte l'Europe centrale. Le stravinskisme étreint des milliers de jeunes musiciens. Mais cette inévitable tyrannie n'empêche point la source indigène de couler en chaque pays sans même qu'il y prenne garde. Elle coule dans les mélodies populaires.

[7] Il n'y a guère plus de cent cinquante ans que les musicologues ont pressenti la valeur ethnographique de ces mélodies, et c'est à peine depuis un demi-siècle que les musiciens de chaque pays vont puiser, dans le folklore ainsi constitué, une inspiration neuve. En France, c'est dans la seconde moitié du XviII siècle que l'on a commencé à réunir de vieilles chansons languedociennes; peu à peu, la recherche s'est étendue à toutes les provinces et, à côté des chercheurs de profession, les poètes et les écrivains n'ont pas été les derniers à seconder leurs efforts, depuis [JeanJacques] Rousseau jusqu'à [Maurice] Bouchor, en passant par George Sand, Gérard de Nerval, Max Buchon et [Jules] Champfleury. En Angleterre, dès 1742, John Parry publiait son Ancient British Music ${ }^{29}$ et des mélodies anglaises, écossaises et gaéliques. Autant en faisait la Scandinavie et autant l'Allemagne.

[8] Les musiciens comprirent l'importance de ces recherches, mais sans les rattacher encore à l'idée nationale. Ils succombaient plutôt à l'attrait exotique des musiques étrangères. De là, dans les Suites du XvIII ${ }^{e}$ siècle, ou dans tant d'œuvres classiques, tant de

28 Couroy cite ici un passage de la Digression sur les anciens et les modernes de l'écrivain et scientifique Bernard de Fontenelle (1657-1757) datant de 1688, dont une deuxième édition paraît en 1708 . Cette remarque de Fontenelle au sujet du climat sert de base au développement de sa pensée sur les différences entre les cultures : «Les différentes idées sont comme des plantes et des fleurs qui ne viennent pas également en toute sorte de climats. Peut-être notre terroir de France n'est-il pas propre pour les raisonnements que font les Égyptiens, non plus que leurs palmiers ; et sans aller si loin, peut-être les orangers qui ne viennent pas aussi facilement ici qu'en Italie, marquent-ils qu'on a en Italie un certain tour d'esprit que l'on n'a pas tout à fait semblable en France. Il est toujours sûr que par l'enchaînement et la réciproque qui est entre toutes les parties du monde matériel, les différences de climats qui se font sentir dans les plantes, doivent s'étendre jusqu'au cerveau et y faire quelque effet » (Fontenelle [1688]1708, p. 204).

29 Il s'agit du premier recueil d'airs gallois (mêlés avec des ballades et chants populaires anglais), publié par le mythique harpiste aveugle gallois John Parry (ca1710-1782) en collaboration avec Evan Williams. 
pièces intitulées a l'ungarese [sic], a la polacca, alla turca. Chopin, en Pologne, paraît être le premier compositeur qui ait senti d'instinct la valeur rénovatrice des chansons nationales. Depuis lors, ont fleuri en chaque pays les compositions de style populaire et, pour ne citer que la France, on connaît assez la floraison bretonne avec [Guy-]Ropartz ou [Paul] Le Flem, vivaraise avec Vincent d'Indy, ou languedocienne avec Déodat de Séverac. C'est pour eux que l'obscur labeur du langage a créé les mots saveur et terroir, pour leurs mélodies où la poésie des ancêtres chatoie sous le réseau harmonique. À l'âme fidèle aux clochers d'enfance, l'esprit vivant apporte des richesses renouvelées sous la poussée des forces séculaires, au fond des provinces silencieuses.

[9] C'est dans ces forces que tous les peuples qui n'ont pas de tradition musicale solide cherchent aujourd'hui leur sauvegarde. Mais un problème ardu se pose à eux. S'il ne s'agissait, pour créer une musique nationale, que de reproduire des chants populaires, la curiosité de l'ethnologue serait satisfaite, mais l'art serait mince (et l'on a n'a que trop souvent l'occasion de le constater). Si le musicien, comme il arrive communément, coule la matière originale dans le moule usé par les âges, il verse dans l'académisme monotone et l'étouffante scholastique ${ }^{30}$.

[10] Les formes traditionnelles doivent lui offrir non un modèle, mais une méthode : à lui de créer des formes, non pas forcément neuves de toutes pièces, mais strictement adaptées à la matière qu'il traite. Cette leçon, un musicien de génie, nourri d'art populaire, la donne à tous aujourd'hui, un créateur qui domine de haut toute la musique contemporaine : Stravinsky.

\section{L'utopie cosmopolite \\ «Projet de centre musical européen " par Arthur et Gertrude Moulton (Le Ménestrel, 1929)}

\section{COMMENTAIRE}

Le projet

Les signataires de cet article paru dans Le Ménestrel du 16 août 1929 sont Arthur Julian Moulton et son épouse, Gertrude Wood Bell Moulton, un couple d'amateurs de musique américains établi dans le Château de la Verrière près de Rambouillet. Le projet de Centre musical européen est vraisemblablement une idée que conçoit Gertrude Moulton dès 1928 et qu'elle se consacrera à réaliser après la séparation du couple en 1929. Si l'article est signé des deux époux alors que les procédures de divorce étaient entamées, c'est parce que le texte en question est une traduction de la brochure explicative qu'ils avaient rédigée autour de 1928 et qu'ils avaient largement fait circuler à travers l'Europe. En effet, des versions traduites en allemand, en italien et en espagnol ont aussi été publiées (Green 2014, p. 179). En plus de la brochure, une maquette illustrant l'ampleur du projet avait aussi été réalisée, fort probablement par Arthur Moulton, qui était particulièrement intéressé par le développement des technologies - il avait installé une panoplie de gadgets électriques dans leur château 
(Birkhead 1934). Les innovations techniques de la scène décrites dans l'article (§ 11), telles que le plancher mobile de la salle autour de la scène et le "service de scène perfectionné " permettant " des effets panoramiques inconnus jusqu'ici », pourraient certainement avoir été pensées par Arthur Moulton.

Dès 1928, le gouvernement français s'était montré intéressé par le projet en offrant un terrain dans le parc de Saint-Cloud. Cependant, avec le krach boursier d'octobre 1929 et la crise économique mondiale qui s'ensuit, le gouvernement abandonne le projet. En partie pour cette raison, Gertrude Moulton quitte la capitale française pour s'établir à Salzbourg et conçoit d'y déménager le Centre musical européen. Son argument principal repose sur l'idée que le centre doit devenir une sorte de Mecque de la musique et que, par conséquent, une métropole commerciale comme Paris serait inappropriée (Green 2014, p. 181). Ces propos font écho à la dénonciation du matérialisme qui est exposée dans la toute première phrase de la brochure datant de 1928, et qui est publiée deux mois avant le krach boursier. Toutefois, c'est la montée du nazisme dans les années 1930 qui empêchera définitivement la fondation du Centre ; Gertrude Moulton semble avoir quitté le continent vers 1937 (ibid., p. 183).

\section{L'utopie cosmopolite}

Le fait que les Moulton désignent Paris comme étant l'endroit idéal pour l'ouverture du Centre musical européen est sans doute motivé par la forte présence d'artistes étrangers à Paris. Ce " cosmopolitisme dans les faits » était par ailleurs à l'origine de nombreuses interventions xénophobes dans la presse musicale ainsi que d'initiatives aux antipodes, dans leur chauvinisme, à celle, cosmopolite, des Moulton - par exemple, la campagne "Pour les artistes français » lancée par Le Guide du concert en 1933 contre la " menace " constituée par le grand nombre de musiciens étrangers à Paris ${ }^{31}$. Loin de considérer cette présence comme une menace, pour les Moulton la capitale française possédait déjà un statut de ville cosmopolite qui favorisait les échanges entre les différentes cultures. Le contexte international promouvant la coopération entre les nations en vue du maintien de la paix dans le monde contribue très certainement à promouvoir les valeurs de l'internationalisme et du cosmopolitisme. Il est toutefois clair que, indépendamment du contexte, le discours des Moulton est clairement inspiré (sinon issu) des idéaux et du langage francs-maçons : l'utopie d'un humanisme cosmopolite basé sur la tolérance, l'image du " triomphe de la lumière cosmique sur les ténèbres et de l'harmonie sur le chaos » (§4), «l'Idée » (ibid.), les caractérisations du Centre musical européen comme "sanctuaire » ( $(5)$ ou "Temple » (\$ 6). Selon les Moulton, l'art est en danger à cause du matérialisme qui conduit les êtres humains à l'arrivisme égoïste et donc à l'individualisme. En revendiquant un échange entre les êtres humains de tous les horizons, "sans distinction de race ni de religion » $(\S 4)$, les auteurs rejettent les conceptions natio-

31 Au sujet de cette campagne ([Bender] 1933) et en général de la xénophobie dans la presse musicale française de l'entre-deux-guerres, voir Lazzaro [à paraître], chap. 5 et 7. 
nalistes et souscrivent à l'utopie d'un endroit où puisse exister une Europe musicale cosmopolite dont les divisions géographiques s'estompent.

\section{L'art comme arme de résistance}

On remarquera l'abondance de termes associés à la lutte dans l'article des Moulton. Ce langage sert à présenter l'art comme une arme contre le mercantilisme jugé " égoïste et cynique » $(\S 1)$. Les deux auteurs ont recours à des termes tels que " résister ", " combattre ", " phalange », " lutter ", " attaquer de front ", " adversaires ", " croisade » ou « citadelle» $(\S 1,2,4,5)$. Dans la critique musicale française contemporaine à la publication de cet article, l'usage d'un tel vocabulaire se trouve habituellement associé à la défense des musiques nationales. Plus particulièrement, c'est le discours visant à distinguer la musique latine de la musique germanique qui utilise cette terminologie, dans le but de mettre en évidence les caractéristiques propres à chacune de ces traditions musicales. Par exemple, dans la conclusion d'un article de 1926 écrit par Pierre-Octave Ferroud, "À propos du Iv festival de la S.I.M.C à Zurich ", on peut lire : «cependant l'intérêt résidait précisément dans la lutte courtoise et féroce que livrèrent les Latins et les Germains - et avec les Germains ceux qui campent sur leur territoire. Car ce fut un combat, et non une pure parade. Chacun resta sur ses positions, et c'est mieux ainsi » (Ferroud 1926, p. 19). Arthur Lourié partage aussi ce discours dans un article de 1931, dans lequel il établit une filiation entre la musique russe et la musique latine. Nous en reproduisons un extrait :

Trois sources : la germanique, la latine, la slave, alimentaient avant la guerre la création musicale.

La musique allemande, le XIX ${ }^{\mathrm{e}}$ siècle durant, défendit une hégémonie à peine contestée. [...]

Vers la fin du siècle, le renouveau national de toutes parts se fit sentir. En pays latin, il partit de France et allait y trouver son apogée en l'art de Claude Debussy. En Russie, le slavisme musical s'était réveillé, et avait suscité, autour de l'auteur de Boris [Godounov], le fameux Groupe des Cinq, dont les aspirations, moins par leur côté slave et national qu'en raison de la jeunesse puissante et de leur richesse en musique inouie, s'allièrent tout naturellement au latinisme naissant pour secouer le joug germanique.

La révolte nationale fut une chose, - la révolte esthétique en fut une autre... (Lourié 1931, p. 160).

C'est une pensée diamétralement opposée que défendent Arthur et Gertrude Moulton dans leur article. L'ennemi visé n'est pas d'origine politique, mais plutôt économique. Cette abstraction du politique dans la diffusion de l'art rappelle les propos tenus en 1923 par Edward J. Dent, président de la SIMC : la Société « n'a été fondée que dans l'intérêt de la musique, et de la musique contemporaine. Elle n'a rien à voir avec la politique ni avec la doctrine sociale de "l'internationalisme" " (Dent 1923, p. 58). Toutefois, on dirait - avec Cœuroy - que toute utopie internationaliste ou cosmopolite est vouée à faire les comptes avec la réalité géopolitique présente et passée : pour pouvoir fonctionner, la SIMC s'organise en sections nationales, et le Centre musical européen prévoit la tenue d'une « saison française en juin, italienne en juillet, etc... » 
(§ 6). Sans être forcément négatif et revendicateur, le discours de ces articles montre néanmoins que les auteurs sont pleinement conscients des différences existant entre les nations. Dans le cas des Moulton, il semble toutefois que le seul critère de division entre les nations dont ils tiennent compte soit la langue parlée : en fait, leurs « saisons » ont le but de proposer des œuvres musicales « chanté[e]s dans le texte original » (ibid.). C'est donc dans le but d'éviter une appropriation linguistique (qui était tout à fait la norme à l'époque) et de valoriser ainsi les versions originales des œuvres que l'idée d'une « saison française en juin, italienne en juillet, etc. » est formulée.

\section{TRANSCRIPTION}

[1] La vague de matérialisme qui déferle actuellement sur le monde a une telle puissance de destruction qu'il paraît impossible aux forces de l'esprit de lui résister. Partout le talent, la pensée pure, l'aspiration désintéressée menacent d'être étouffés par un mercantilisme égoïste et cynique.

[2] Or, l'art créateur a toujours été l'une des armes les plus efficaces données à l'homme pour résister aux assauts du matérialisme et pour combattre ses instincts inférieurs. Les dons de l'esprit qui ennoblissent notre vie sous la forme de culture, de science, d'art ou de religion sont, en définitive, les seuls phares qui éclairent notre route.

[3] Les empires s'effondrent, les hommes périssent. Seules, les créations de l'esprit résistent au temps et la flamme des grandes œuvres inspirées se transmet d'âge en âge comme une torche sacrée.

[4] Il est indispensable aujourd'hui que les hommes désireux de porter ce flambeau et de ranimer la flamme s'unissent dans un effort universel pour la réalisation des idéals communs à toute l'humanité civilisée. Cette phalange, groupée sans distinction de race ni de religion, travaillera à l'avènement de la paix mondiale en s'efforçant de développer entre les peuples la compréhension et la tolérance. Elle ranimera la foi de l'humanité troublée dans le triomphe de la lumière cosmique sur les ténèbres et de l'harmonie du chaos. Elle partagera le résultat de son labeur avec ses semblables afin que tous les hommes - individuellement et collectivement - participent dans la plus large mesure à l'effort sublime. Les penseurs du monde entier sentent que l'heure est venue de lutter contre le danger menaçant et de combattre le matérialisme sous toutes ses formes. Il s'agit de sauver l'humanité des forces qui la dominent à l'heure actuelle et la feraient glisser vers un plan de médiocrité et d'agitation stérile pour la conduire finalement au suicide moral. Au lieu d'accepter, comme un mal inévitable, l'état de turpitude actuelle, nous devons attaquer de front les adversaires de l'Idée : l'arrivisme égoïste, les jalousies mesquines, l'âpreté financière, la poursuite éperdue des possessions matérielles et proclamer cette vérité, que c'est en donnant tout que nous gagnons tout. L'Homme ne peut se satisfaire longtemps des joies stériles que procurent les biens périssables. Il s'agit une fois de plus de «nous arracher aux mornes nonchalances » et de partir pour une nouvelle croisade.

[5] Dans ce but, nous proposons de créer un centre artistique, un sanctuaire où le rêve et l'inspiration puissent s'épanouir dans une atmosphère favorable, une citadelle contre les remparts de laquelle les forces néfastes viennent se briser... Ce sera un foyer d'art pur où, au lieu d'ambitions égoïstes, nous verrons des efforts coordonnés vers un but altruiste et où la routine paralysant l'élan sera remplacée par une spontanéité qui permettra aux personnalités de se manifester dans toute leur énergie et leur force créatrice. Il existe des musées et des bibliothèques où nous conservons les œuvres immortelles du passé et du présent pour les mettre à la portée de tous, mais l'art plus insaisissable et subtil de la musique est privé de temples dignes de lui. 
[6] Le projet serait d'élever aux environs de Paris un Temple de la musique où tous les chefs-d'œuvre classiques et modernes puissent être exécutés en été, chantés dans le texte original. (Par exemple, il pourrait y avoir une saison française en juin, italienne en juillet, etc., avec quatre à six représentations par semaine et les autres jours, des concerts symphoniques ou des récitals privés.)

[7] Un projet de ce genre a déjà été conçu et réalisé par Wagner à Bayreuth, mais seulement pour l'exécution de ses propres œuvres. Cette fois, il s'agirait d'un rêve beaucoup plus vaste et d'un programme très éclectique.

[8] Il faudrait trouver pour ce temple un site assez proche de Paris pour être d'accès facile et en même temps assez éloigné du bruit et de l'agitation de la capitale pour offrir le cadre naturel pittoresque et l'atmosphère de recueillement nécessaires pour l'audition et l'exécution des chefs-d'œuvre de la musique. Il est inutile de dire que ce théâtre, loin de nuire en aucune façon à l'Opéra, lui servirait au contraire de complément et ferait de Paris, en été, un but de pèlerinage musical.

[9] Ce projet est conçu sur un plan très vaste, car, en plus du théâtre, il y aurait une école de musique où de jeunes artistes pourraient venir parachever leur éducation. Il faudrait donc ajouter au bâtiment principal des studios et des locaux d'habitation, restaurant, etc. pour les élèves ainsi que pour le personnel du théâtre. Les mois d'hiver seraient employés à l'étude du répertoire, étude poussée vers la plus parfaite réalisation artistique possible. Cette école attirerait à Paris les jeunes artistes du monde entier : cette ville étant déjà un grand centre d'art, ces jeunes gens auraient, une fois leur éducation terminée, l'occasion de se faire entendre par le plus compréhensif et le plus cultivé des publics. Les études seraient payantes, mais il y aurait un certain nombre de places réservées aux élèves boursiers.

[10] Bien entendu, cette entreprise ne doit poursuivre qu'un but artistique : celui de relever le niveau de l'art musical. Après un examen impartial des différents théâtres d'opéras, des sociétés de concerts, etc., il est évident que cet art est actuellement en décadence, d'abord pour les raisons de mercantilisme indiquées plus haut et ensuite, parce qu'il est étouffé par une tradition surannée. Il s'agit donc non pas de faire de l'argent, mais de l'art, en offrant au public les meilleures auditions musicales aux plus bas prix, dans un cadre harmonieux où soient utilisés, pour les décors et la mise en scène, les derniers perfectionnements dus à la science moderne.

[11] Dans ce but, des plans ont été dessinés et une maquette exécutée indiquant toutes les innovations. Un service de scène perfectionné permettrait de rapides changements de décor ${ }^{32}$ et des effets panoramiques inconnus jusqu'ici. En outre, la dimension de la scène offrirait la possibilité de représenter de vastes tableaux ; enfin, au lieu d'une scène mobile, le plancher du pourtour serait mobile. Un théâtre en plein air (théâtre de verdure) ayant un décor naturel, un lac, des collines, des rochers, un jardin pourrait également être utilisé dans certains cas.

[12] On estime que la réalisation de ce projet coûterait de 4 à 6 millions de dollars. $\mathrm{Au}$ lieu d'avoir recours à une souscription publique, il serait préférable que cette somme fût fournie par un ou deux donateurs. Tel a été le cas lors de la création des bibliothèques gratuites par André Carnegie ${ }^{33}$ dont le but fut absolument altruiste et désintéressé.

32 Les Moulton écrivent « décors".

33 Les auteurs font référence à Andrew Carnegie (1835-1919), dont les œuvres philanthropiques ont permis la fondation de nombreuses bibliothèques. Nous ajouterons que Carnegie est aussi l'instigateur d'un mouvement pour la paix en 1910, le Carnegie Endowment for International Peace qui pourrait avoir inspiré Arthur et Gertrude Moulton. 
De même, maintenant, devons-nous trouver le mécène dont la générosité et la compréhension permettront à l'art musical de s'épanouir librement. Il faut le sauver du danger actuel qui prostitue le talent, entrave le progrès et étouffe l'inspiration.

\section{BIBLIOGRAPHIE ${ }^{34}$}

Balzac, Honoré de ([1830]1838), Physiologie du mariage, ou Méditations de philosophie éclectique sur le bonheur et le malheur conjugal, 2e éd., Paris, Charpentier. Disponible sur Gallica : http://gallica.bnf. fr/ark:/12148/bpt6k96682003.

B[ender],G[abriel] (1933), « Pour les artistes français », Le Guide du concert, vol. 20, n 5 (3 novembre), p. 107.

Bernard, Robert (1930), Les tendances de la musique française moderne. Cours d'esthétique, huit conférences prononcées au Conservatoire international de Paris et en Sorbonne, Paris, Durand / Genève, Revue mensuelle.

Bernard, Robert (1938), "Les caractéristiques de la musique française ", La Revue musicale, vol. 20, $\mathrm{n}^{\circ} 181$ (février), p. 120-131. Disponible dans la banque de données du PHEM : http://lmhsbd.oicrm. org/media/ART-BER-1938-02.pdf.

Bernard, Robert (1946), « Nationalisme et internationalisme artistiques (à propos des programmes des concerts) », La Revue musicale, n 198 (mars), p. 46-49.

Birkhead, Mark (1934) "Arthur Moulton Weds at His Château », Chicago Sunday Tribune (12 août), partie 8, p. 3.

Bouyer, Raymond (1905), "Kapellmeister d'outre-monts (Petites notes sans portée) ", Le Ménestrel, vol. $71, \mathrm{n}^{\circ}$ 5/3853 (29 janvier), p. 35-36. Disponible dans la banque de données du PHEM : http://lmhsbd.oicrm.org/media/ART-BORa-1905-01.pdf.

Collet, Henri (1919), «L'internationalisme musical», Le Courrier musical, vol. 21, n 20 (15 décembre), p. 307-308. Disponible dans la banque de données du PHEM : http://1mhsbd.oicrm.org/media/ ART-COHa-1919-01.pdf.

Constant, Benjamin (1809), Wallstein, tragédie en 5 actes, Paris, Paschoud. Disponible sur Gallica : http://gallica.bnf.fr/ark:/12148/bpt6k6498986r.

Couroy, André [Jean Belime] ([1928a]1930), Panorama de la musique contemporaine, Paris, Kra ; plusieurs éditions revues et augmentées parues en 1928 et 1930.

Couroy, André [Jean Belime] ([1928b]), « Sous le signe du national », Le Ménestrel, vol. 90, n 5/4788 (3 février), p. 45-46. Il s'agit de l'introduction à Couroy [1928a]1930. Disponible dans la banque de données du PHEM : http://1mhsbd.oicrm.org/media/ART-COA-1928-02.pdf.

Dent, Edward (1923), «Internationalisme et musique », La Revue musicale, vol. 4 n ${ }^{\circ} 10$ (août), p. 5860. Disponible dans la banque de données du PHEM : http://lmhsbd.oicrm.org/media/ARTDEEb-1923-01.pdf.

Estève, Joseph (1939), «L'internationalisme et la musique », La Revue musicale, vol. 20, nº 190 (avril), p. 170-176. Disponible dans la banque de données du PHEM : http://lmhsbd.oicrm.org/media/ ART-ESJ-1939-01.pdf.

Ferroud, Pierre-Octave (1926), «À propos du Iv ${ }^{e}$ Festival de la S.I.M.C. à Zurich ", Revue Pleyel, n 34 (15 juillet), p. 17-19. Disponible dans la banque de données du PHEM : http://lmhsbd.oicrm.org/ media/ART-FEP-1926-01.pdf. 
Fontenelle, Bernard de ([1688]1708), Poésies pastorales. Avec un traité sur la nature de l'églogue et une digression sur les anciens et les modernes, troisième édition augmentée, Paris, Michel Brunet. Disponible sur Gallica : http://gallica.bnf.fr/ark:/12148/bpt6k97390056.

Fornerod, Aloys (1928), [réponse à l'enquête], Musique, vol. 2, no 1 (15 octobre), p. 595-586. Disponible dans la banque de données du PHEM : http://1mhsbd.oicrm.org/media/ART-FOA-1928-02.pdf.

Gil-Marchex, Henri (1923), «Le Festival de musique contemporaine de Salzbourg », Le Monde musical, vol. $34, \mathrm{n}^{\text {os }}$ 15-16 (août), p. 270-271.

Gil-Marchex, Henri (1924), «Le Festival de Salzbourg », Le Monde musical, vol. 35, nºs 15-16 (juilletaoût), p. 285.

Green, Nancy L. (2014), Les Américains de Paris. Hommes d'affaires, comtesses et jeunes oisifs, 1880-1941, traduit de l'américain par Patrick Hersant, Paris, Belin.

Gumplowicz, Philippe (2004), « Musicographes réactionnaires des années 1930 », Le Mouvement social, année 2004, no 3 (n 208$)$, p. 91-124.

Haefeli, Anton (1982), IGNM, Die Internationale Gesellschaft für Neue Musik. Ihre Geschichte von 1922 bis zur Gegenwart, Zürich, Atlantis.

Hoérée, Arthur (1926), "Querelles esthétiques. La Musique, langage proprement national : en marge du festival de Zurich », Revue Pleyel, $\mathrm{n}^{\circ} 35$ (15 août), p. 17-20. Disponible dans la banque de données du PHEM : http://lmhsbd.oicrm.org/media/ART-HOA-1926-02.pdf.

Hoérée, Arthur (1928), "Arnold Schœnberg à Paris », Musique, vol. 1, n 5 (15 février), p. 214223. Disponible dans la banque de données du PHEM : http://lmhsbd.oicrm.org/media/ARTHOA-1928-07.pdf.

Hoérée, Arthur (1938), "Cours d'esthétique par Robert Bernard (Schola Cantorum) ", La Revue musicale, vol. $19, \mathrm{n}^{\circ} 182$ ( $1^{\text {er }}$ mars), p. 226. Disponible dans la banque de données du PHEM : http://lmhsbd.oicrm.org/media/ART-HOA-1938-01.pdf.

Iriye, Akira (2000), Cultural Internationalism and World Order, Baltimore, Johns Hopkins University Press.

Iriye, Akira (2002), Global Community. The Role of International Organizations in the Making of the Contemporary World, Berkeley/Los Angeles, University of California Press.

Jean-Aubry, G. [Jean-Frédéric-Émile Aubry] (1922), La musique et les nations, Paris, Éditions de la Sirène / Londres, Chester.

Joye, Sylvie (2016), «Représentations modernes et contemporaines. Barbares redécouverts, barbarie réinventée ", dans Bruno Dumézil (dir.), Les barbares, Paris, Presses universitaires de France, p. 89-116.

Kalomiris, Manolis (1924), " [Consultation sur la musique contemporaine] ", Le Courrier musical et théatral, vol. 26, $\mathrm{n}^{\circ} 1-2$ (1 $1^{\text {er }}$ janvier), p. 14. Disponible dans la banque de données du PHEM : http://1mhsbd.oicrm.org/media/ART-KAM-1924-01.pdf.

La Laurencie, Lionel de (1920), "L'exotisme dans l'ancienne musique française ", Le Courrier musical, vol. 22, no 4 (15 février), p. 61-63. Disponible dans la banque de données du PHEM : http://1mhsbd.oicrm.org/media/ART-LALa-1920-01.pdf.

Landry, Lionel (1927), La sensibilité musicale. Ses éléments, sa formation, Paris, Félix Alcan. Disponible sur Echo : http://echo.mpiwg-berlin.mpg.de/MPIWG:R1NGNY66.

Lazzaro, Federico [à paraître], Écoles de Paris en musique, 1902-1950. Identités, nationalisme, cosmopolitisme, Paris, Vrin.

Lourié, Arthur (1931), "Perspectives de l'École Russe ", La Revue musicale, vol. 12, n 117-118 : Géographie musicale 1931 (juillet-août), p. 160-165. Disponible dans la banque de données du PHEM : http://lmhsbd.oicrm.org/media/ART-LOAa-1931-02.pdf.

Margat, Yves (1935), "Variations... sans thème (Harmonioso) ", Le Guide du concert, vol. 21, $\mathrm{n}^{\circ}$ 27-28-29 (5 avril), p. 716. Disponible dans la banque de données du PHEM : http://lmhsbd.oicrm.org/media/ART-MAY-1935-02.pdf. 
Migot, Georges (1924), "La musique et l'hospitalité française », Revue Pleyel, no 6 (15 mars), p. 1516. Disponible dans la banque de données du PHEM : http://lmhsbd.oicrm.org/media/ARTMIGa-1924-02.pdf.

Migot, Georges (1928), [réponse à l'enquête], Musique, vol. 1, $\mathrm{n}^{\text {os }} 11-12$ (15 septembre), p. 500509. Disponible dans la banque de données du PHEM : http://lmhsbd.oicrm.org/media/ARTMIGa-1928-02.pdf.

Moulton, Arthur, et Gertrude Moulton (1929), "Projet de centre musical européen ", Le Ménestrel, vol. 91, $\mathrm{n}^{\circ} 33$ (16 août), p. 367-368. Disponible dans la banque de données du PHEM : http://lmhsbd.oicrm.org/media/ART-MOAb-1929-01.pdf.

Pénisson, Pierre (dir.) (1995), De l'universalité européenne de la langue française, 1784, Paris, Fayard.

Rivarol, Antoine de ([1784]1785), De l'universalité de la langue française, $2^{\mathrm{e}}$ éd., Berlin et Paris, Bailly et Dessenne. Disponible sur Gallica : http://gallica.bnf.fr/ark:/12148/bpt6k63229414.

Autres éditions consultées :

1929 : notices et notes par Albert Cherel, Paris, Hatier.

1936 : avec une notice biographique, une notice historique et littéraire, des notes explicatives, des jugements, un questionnaire sur le discours et des sujets de devoirs par Maurice Favergeat, Paris, Larousse.

1991 : [Paris], Obsidiane/Bibliothèque nationale.

Roland-Manuel [Roland Alexis Manuel Lévy] (1925), "André Messager et "le retour à la rose" ", Revue Pleyel, $\mathrm{n}^{\circ} 27$ (15 décembre), p. 8-10. Disponible dans la banque de données du PHEM : http://lmhsbd.oicrm.org/media/ART-LER-1925-10.pdf.

Roland-Manuel [Roland Alexis Manuel Lévy] (1928), "Coup d'œil sur la saison », Musique, vol. 1, n 9 (15 juin), p. 389-392. Disponible dans la banque de données du PHEM : http://lmhsbd.oicrm.org/ media/ART-LER-1928-03.pdf.

Schwab, Johann Christoph ([1803]2005), Dissertation sur les causes de l'universalité de la langue françoise et la durée vraisemblable de son empire [Über die Ursachen der Allgemeinheit der französischen Sprache und der wahrscheinlichen Dauer ihrer Herrschaft], traduit de l'allemand par Denis Robelot, Pary, Lamy. Disponible sur Gallica : http://gallica.bnf.fr/ark:/12148/bpt6k5580077z. Édition moderne présentée par Freeman G. Henry, Amsterdam, Rodopi, 2005.

Sluga, Glenda (2013), Internationalism in the Age of Nationalism, Philadelphia, University of Pennsylvania Press.

Stoecklin, Paul de (1919), «La délivrance », Le Courrier musical, vol. 21, nº 12 (15 juin), p. 177-178.

Vuillemin, Louis (1923), « Musique et nationalisme (Notes sans mesure) », Le Courrier musical, vol. 25, $\mathrm{n}^{\circ} 4$ (15 février), p. 65. Disponible dans la banque de données du PHEM : http://1mhsbd.oicrm.org/ media/ART-VUL-1923-01.pdf. 